\title{
The South American Physical Activity and Sedentary Behavior Network (SAPASEN)
}

\author{
André O. Werneck ${ }^{1}$ (D), Se-Sergio Baldew ${ }^{2}$, J. Jaime Miranda ${ }^{3}$, \\ Óscar Incarbone ${ }^{4}$ and Danilo R. Silva ${ }^{5}$; South American Physical \\ Activity and Sedentary Behavior Network Collaborators
}

\begin{abstract}
The present article describes the South American Physical Activity and Sedentary Behavior Network, which was designed to provide ongoing transnational empirical evidence about physical activity and sedentary behavior in South America. The first goal of this initiative was to form a representative body of researchers and policy makers from all South American countries (Argentina, Bolivia, Brazil, Chile, Colombia, Ecuador, Guyana, Paraguay, Peru, Suriname, Uruguay and Venezuela) to establish priorities and targets for the short, medium and long term. Examples are given of connecting physical activity and sedentary data from existing surveys in several of the partner countries. The main objective of the South American Physical Activity and Sedentary Behavior Network will be to impact policies on physical activity and sedentary behavior in South America according to the singularities of each country or region. By encouraging an inclusive and collaborative effort, we expect that the South American Physical Activity and Sedentary Behavior Network will support the connection between researchers from South America as well as provide a better comprehension of the epidemiology of physical activity and sedentary behavior regionally.
\end{abstract}

Keywords: Exercise, adults, sedentary lifestyle

\section{Introduction}

Physical activity, defined as any bodily movement produced by skeletal muscles that requires energy expenditure (1), is well established as a major protective factor for health. It is estimated that physical inactivity, which is the lack of sufficient levels of physical activity according to recommendations from the World Health Organization (2), is responsible for $7.1 \%$ of coronary heart diseases, $8.7 \%$ of type 2 diabetes and $11.4 \%$ of all-cause mortality among Latin Americans. Thus, if we eliminate physical inactivity, life expectancy would increase by 0.8 years (3). Unfortunately, physical inactivity is still highly

1. Scientific Research Group Related to Physical Activity (GICRAF), Laboratory of Investigation in Exercise (LIVE), Department of Physical Education, São Paulo State University (UNESP), Presidente Prudente, Brazil.

2. Department of Physical Therapy, Faculty of Medical Sciences, Anton de Kom University of Suriname, Paramaribo, Suriname.

3. CRONICAS Centre of Excellence in Chronic Diseases, and Facultad de Medicina "Alberto Hurtado," Universidad Peruana Cayetano Heredia, Lima, Peru.

4. Department of health, nutrition and physical activity, ISALUD university, Buenos Aires, Argentina.

5. Department of Physical Education, Federal University of Sergipe - UFS, São Cristóvão, Brazil.

Correspondence to: André O. Werneck, Department of Physical Education, São Paulo State University (UNESP), Rua Roberto Símonsen, 305. Presidente Prudente, São Paulo, 19060-900 Brazil. Email: andreowerneck@gmail.com. Phone number: +55 $183229-5388$

(This manuscript was submitted on 6 January 2019. Following blind peer review, it was accepted for publication on 27 May 2019)

Global Health Promotion 1757-9759; Vol 0(0): 1-6; 859577 Copyright (C) The Author(s) 2019, Reprints and permissions: http://www.sagepub.co.uk/journalsPermissions.nav DOI: 10.1177/1757975919859577 journals.sagepub.com/home/ghp 
prevalent worldwide (4). Specifically, in South America, estimates revealed that physical inactivity is around $31 \%$ (4), generating additional costs to national health plans. Similarly, an independent dimension of human behavior, sedentary behavior (5) is receiving increasing attention due to its impact on metabolic, cardiovascular and mental health $(6,7)$. It is estimated that sedentary behavior, defined as any waking behavior characterized by energy expenditure $\leqslant 1.5$ metabolic equivalents while in a sitting or reclining posture (5), is responsible for about $3.2 \%$ of all-cause mortality in Latin America (6).

\section{International initiatives for physical activity and sedentary behavior surveillance}

In the face of these public health concerns, several transnational actions are being developed, aiming to stimulate physical activity, but not to directly decrease sedentary behavior, worldwide. The World Health Organization has provided recommendations for physical activity practice since 2010 and recently launched 'The global action plan on physical activity 2018 - 2030', which includes milestones for the reduction of physical inactivity around the world (8). Moreover, The Lancet launched two physical activity series (2012 and 2016) and the Lancet Physical Activity Observatory, which aims to assess physical activity worldwide (9). In order to create the 'Global Observatory for Physical Activity (GoPA!) (Derived from Lancet Physical Activity Observatory), country report cards' with the prevalence of physical activity within each country, as well as the number of active researchers, existence of physical activity national plans and periodic national surveillance of physical activity, are required (10). Concerning sedentary behavior, the scenario is different. As the study of sedentary behavior is newer than physical activity, global plans are not well developed. Globally, the Sedentary Behavior Research Network is the world's greatest plan, which primarily focuses on the creation of a final consensus of terminologies about sedentary behavior (5) as well as the dissemination of scientific evidence in this field. However, to the best of our knowledge, there are no specific global plans aiming at sedentary behavior surveillance to help combat this negative exposure. Recently, GoPA! also started an effort for the inclusion of sedentary behavior indicators in the report cards, but it still in initial phase.

\section{South American initiatives for physical activity and sedentary behavior}

In South America, which hosts 0.9 billion people and has different cultures and different patterns of disease across and within countries, there are two major projects aiming to promote physical activity. The first, the 'AGITA' program, was created to promote the recommendation of physical activity, with repercussions inside Brazil as well as globally, including other countries in South America. The 'Red de Actividad Física de las Américas' (RAFA/PANA), for example, was derived from AGITA, also to promote physical activity in South America, through conferences, dissemination of evidence and integrating research to policy actions. The second, the 'GUIA project', aimed to promote physical activity in Latin America (11), specifically in Colombia and Brazil. GUIA evaluated two interventions in Brazil, provided systematic reviews and stimulated the interlocution between research and policy (12). Even with the above-mentioned actions in Latin America, there is no transnational initiative aiming to provide surveillance of physical activity and sedentary behavior and their determinants. Given the social, cultural and economic diversity between and within Latin American countries, it is also expected that different correlates and determinants of physical activity and sedentary behavior are present. Thus, effective interventions aiming to promote health behaviors could vary widely.

\section{The SAPASEN project}

The South American Physical Activity and Sedentary Behavior Network (SAPASEN) was designed to provide ongoing transnational empirical evidence about physical activity and sedentary behavior in South America, by evaluating the prevalence, distribution and correlates/determinants of these behaviors. The first step in setting up the network was to form a representative body of researchers and policy makers from all South American countries (Argentina, Bolivia, Brazil, Chile, Colombia, Ecuador, Guyana, Paraguay, Peru, Suriname, Uruguay and Venezuela) in order to collectively establish priorities and short, medium and long-term goals. 
In the short term, SAPASEN will explore epidemiological data on physical activity and sedentary behavior using national representative datasets from South American countries and describe the prevalence of each behavior, sociodemographic distribution, possible determinants of these behaviors as well as possible consequences, such as chronic diseases and mental health. SAPASEN also aims to investigate the methods of data collection in each country.

After the first period, the medium-term priority will be to standardize the collecting methods in the national health surveys, including physical activity and sedentary behavior questionnaires, stimulating the communication with national health agencies of each country. Therefore, SAPASEN aims to provide continuous information on physical activity and sedentary behavior prevalence over the years, as well as to stimulate community interventions, considering regional specificities. For this, national surveys with information on physical activity and sedentary behavior (including total sitting time and screen time measures), as well as sociodemographic factors, environmental and negative health outcomes (including chronic diseases such as hypertension, type 2 diabetes and mental health) conducted among adults between 18 and 59 years from South American countries (Argentina, Bolivia, Brazil, Chile, Colombia, Ecuador, Guyana, Paraguay, Peru, Suriname, Uruguay and Venezuela) will be analyzed. Finally, by also exploring data on sitting time and screen time, including TV viewing as part of national surveys from South American countries, SAPASEN will aim to fill the gap of missing surveillance data on sedentary behavior from a transnational and regional level.

\section{Barriers and supporting factors for SAPASEN}

One of the greatest barriers for SAPASEN will be to initiate communication with each government in South America and enlighten them about the importance of standardization of physical activity / sedentary behavior collection methods, especially among countries passing through economic and/or political crisis and consequently promoting priorities other than public health. Political transitions and changes will also present challenges for the SAPASEN actions.

Specifically, given the initial aim of SAPASEN, the first barrier will be to harmonize data from different countries, considering the inclusion of different data collection methods adopted in different countries. Similarly, another challenge will be the sensitization of each country regarding the adoption of standardized methods in their national health surveys, especially concerning physical activity and sedentary behavior. SAPASEN will look for funding opportunities aiming to perpetuate continuous surveillance in South America. It is our hope that the collaboration of researchers and policy makers combined in SAPASEN as well as the appeal for the surveillance of risk/protective behaviors should reduce these barriers.

\section{Preliminary data}

To begin addressing SAPASEN objectives, we conducted analyses of physical activity and sedentary behavior prevalence using already existing nationally representative datasets. National surveys from Argentina (Encuesta Nacional de Factores de Riesgo 2013), Brazil (Pesquisa Nacional de Saúde 2013), Chile (Encuesta Nacional de Salud 2009-2010), Ecuador (Encuesta Nacional de Salud y Nutrición 2012), Peru (Encuesta Nacional de Hogares, Módulo de Mediciones Antropométricas, 2011), and Suriname (The Suriname Health Study, 2013) were harmonized for a prevalence analysis. In the initial phase of SAPASEN, we also identified that Colombia, Paraguay and Venezuela have national surveys with physical activity data. However, Paraguay and Venezuela did not provide data and Colombia did not reply on the invitation to be part of SAPASEN. All samples were calculated through complex sampling. Data of physical activity and sedentary behavior of 117,014 adults (Argentina $=26,932$; Brazil $=$ 52,490; Chile $=3719$; Ecuador $=19,883$; Peru $=$ 8820 ; Suriname $=5170$ ) were analyzed using sampling weights for each country.

National surveys adopted different questionnaires to assess the physical activity and sedentary behavior. For the current study, we analyzed total physical activity (sum of leisure, transportation and occupational domains) and two indicators of sedentary behavior (TV viewing and sitting time). Surveys from Argentina and Ecuador did not include occupational physical activity, and surveys from Ecuador and Brazil did not include sitting time. The Brazilian survey included total TV viewing instead of sitting time.

Figure 1 shows the prevalence of more than 150 minutes of moderate-to-vigorous physical 
A. O. Werneck et al.

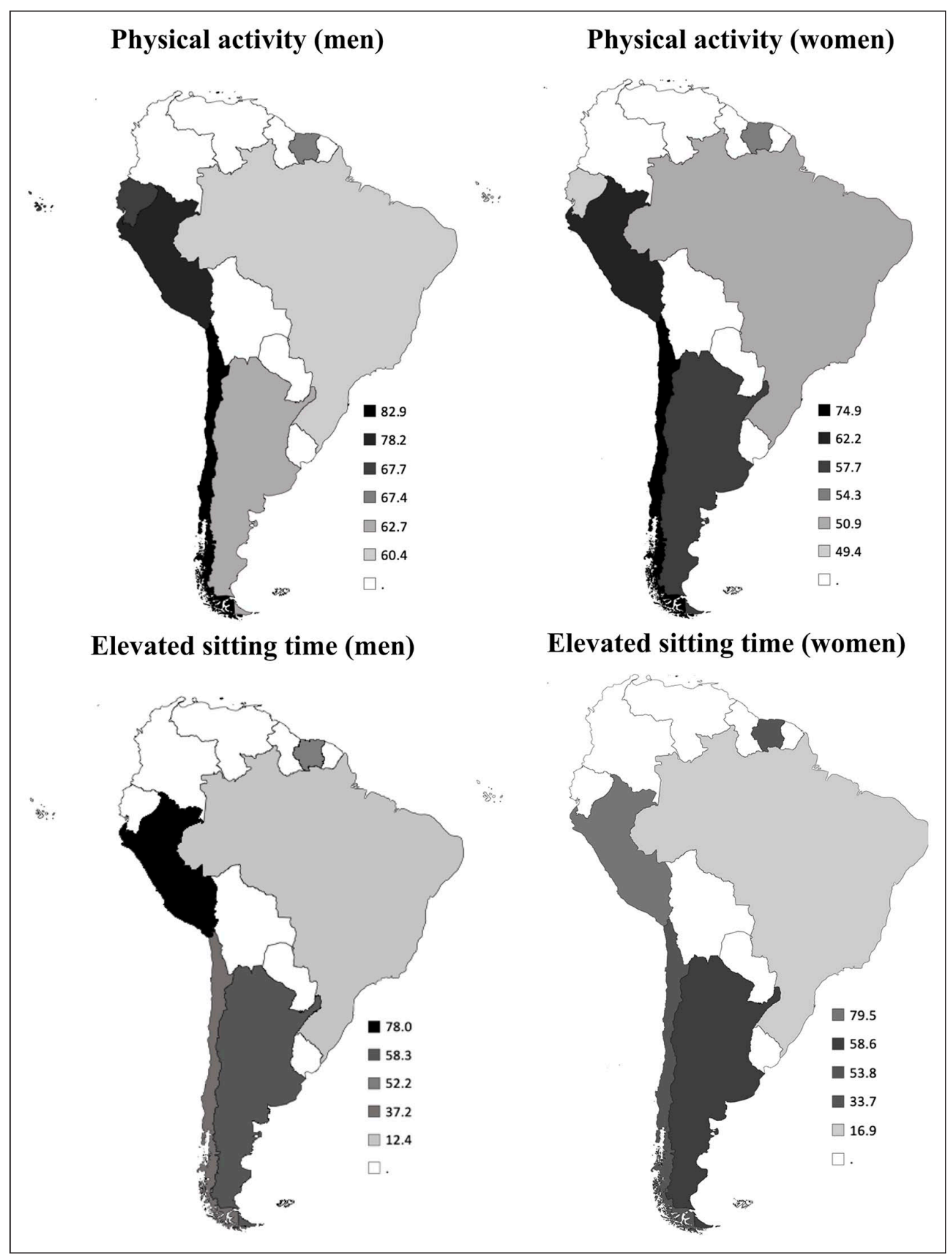

Figure 1. Prevalence of at least $150 \mathrm{~min} /$ week of habitual physical activity and more than $4 \mathrm{~h} / \mathrm{day}$ of sitting time in males and females from SAPASEN.

Note: Data are presented using frequencies. 
activity per week and more than 4 hours/day of sedentary behavior by sex and country. The prevalence of physical activity ranged between $60.4 \%$ (95\% CI: 59.3 to 61.5 ) (Brazil) and $82.9 \%$ (95\% CI: 79.7 to 85.7 ) (Chile) among men, and between $49.4 \%$ (95\% CI: 47.7 to 51.0 ) (Ecuador) and $74.9 \%$ (95\% CI: 71.7 to 77.7 ) (Chile) among women. Regarding sitting time, prevalence of more than 4 hours/day ranged between $78.0 \%$ (95\% CI: 76.3 to 79.6 ) (Peru) and 37.2\% (95\% CI: 33.3 to 41.3 ) (Chile) among men, and between $79.5 \%$ (95\% CI: 78.0 to 80.9 ) (Peru) and $33.7 \%$ (95\% CI: 30.6 to 37.1 ) (Chile). In Brazil, $12.4 \%$ (95\% CI: 11.7 to 13.1 ) of men and $16.9 \%$ (95\% CI: 16.2 to 17.7 ) of women reported more than $4 \mathrm{~h} /$ day in TV viewing.

The value of the SAPASEN approach is illustrated through these preliminary results, and already indicate potential priority areas for interventions in Brazil and Ecuador for physical activity, and in Peru for sitting time. The next steps of SAPASEN can help to further clarify potential determinants of both behaviors and how they can vary across South American countries. These efforts also signal to the need for a standardized physical activity collection method for South American countries.

Once a solid empirical base is established, SAPASEN hopes to provide information to impact policies on physical activity and sedentary behavior in South America according to the specificities of each country or region. We also expect that SAPASEN can encourage an inclusive and collaborative international effort by supporting the connection between researchers from South America, as well as providing a better comprehension of the epidemiology of physical activity and sedentary behavior regionally.

\section{SAPASEN collaborators}

Jaime Leppe Zamora (Facultad de Medicina Clinica Alemana Universidad del Desarrollo, Santiago, Chile), Enrique Chavez Cevallos (Universidad de las Fuerzas Armadas ESPE. Ecuador), William Adu-Krow (PAHO/ WHO Representation, Guyana), Guillermo Sequera (Ministerio de Salud Pública y Bienestar Social, Paraguay), Rodrigo Carrillo-Larco (Universidad Peruana Cayetano Heredia, Lima, Peru), Bastiaan de Boer (Suriname representation), Oscar Díaz Arnesto (Uruguay representation), Connie Garcia (Facultad de Medicina UCV, Caracas, Venezuela), Luis Fernando Leanes (PAHO/WHO Representation, Bolívia).

\section{Authors' contributions}

AOW and DRS: literature search, study design, and writing. SB, JJM and OI: study design and revision of the first draft.

\section{Ethics committee approval}

Not applicable.

\section{Declaration of conflicting interest}

The authors declare that there is no conflict of interest.

\section{Funding}

André O. Werneck is supported by the São Paulo Research Foundation with a master's degree scholarship (FAPESP process: $2017 / 27234-2$ )

\section{ORCID iD}

André O. Werneck iD https://orcid.org/0000-0002-9166-4376

\section{References}

1. Caspersen CJ, Powell KE, Christenson GM. Physical activity, exercise, and physical fitness: definitions and distinctions for health-related research. Public Health Rep. 1985; 100: 126-131.

2. World Health Organization. Global Recommendations on Physical Activity for Health. Geneva, Switzerland: World Health Organization; 2010.

3. Lee IM, Shiroma EJ, Lobelo F, Puska P, Blair SN, Katzmarzyk PT, et al. Effect of physical inactivity on major non-communicable diseases worldwide: an analysis of burden of disease and life expectancy. Lancet. 2012; 380: 219-229.

4. Sallis JF, Bull F, Guthold R, Heath GW, Inoue S, Kelly $\mathrm{P}$, et al. Progress in physical activity over the Olympic quadrennium. Lancet. 2016; 388: 1325-1336.

5. Tremblay MS, Aubert S, Barnes JD, Saunders TJ, Carson V, Latimer-Cheung AE, et al. Sedentary behavior research network (SBRN) - terminology consensus project process and outcome. Int J Behav Nutr Phys Act. 2017; 14: 75.

6. Rezende LFM, Sá TH, Mielke GI, Viscondi JYK, ReyLópez JP, Garcia LMT. All-cause mortality attributable to sitting time: analysis of 54 countries worldwide. Am J Prev Med. 2016; 51: 253-263.

7. Vancampfort D, Firth J, Schuch FB, Rosenbaum S, Mugisha J, Hallgren M, et al. Sedentary behavior and physical activity levels in people with schizophrenia, bipolar disorder and major depressive disorder: a global systematic review and meta-analysis. World Psychiatry. 2017; 16: 308-315.

8. World Health Organization. Global Action Plan on Physical Activity 2018-2030: More Active People for a Healthier World. Geneva, Switzerland: World Health Organization; 2018. 
9. Hallal PC, Martins RC, Ramírez A. The lancet physical activity observatory: promoting physical activity worldwide. Lancet. 2014; 384: 471-472.

10. Ramirez Varela A, Salvo D, Pratt M, Milton K, Siefken K, Bauman A, et al. Worldwide use of the first set of physical activity Country Cards: the Global Observatory for Physical Activity - GoPA! Int J Behav Nutr Phys Act. 2018; 15: 29.
11. Matsudo VKR, Lambert EV. Bright spots, physical activity investments that work: Agita Mundo global network. Br J Sports Med. 2017; 51: 1382-1383.

12. Pratt M, Brownson RC, Ramos LR, Malta DC, Hallal PC, Reis RS, et al. Project GUIA: a model for understanding and promoting physical activity in Brazil and Latin America. J Phys Act Health. 2010; 7(Suppl 2): S131-S134. 